Knauf, Rainer; Sakurai, Yoshitaka ; Tsuruta, Setsuo :

Toward making didactics a subject of knowledge engineering

Zuerst erschienen in:

Proceedings // The Seventh IEEE International Conference on Advanced Learning Technologies, ICALT 2007 : 18 - 20 July 2007, Niigata, Japan / sponsored by IEEE Technical Committee on Learning Technology ... - Los Alamitos, Calif. [u.a.] : IEEE Computer Society, ISBN 0-7695-2916-X=978-0-7695-2916-5,

S. 788-792.

DOI 10.1109/ICALT.2007.259 


\title{
Toward Making Didactics a Subject of Knowledge Engineering
}

\author{
Rainer Knauf, Yoshitaka Sakurai and Setsuo Tsuruta \\ Technical University Ilmenau, Germany, Tokyo Denki University, Japan \\ rainer.knauf@tu-ilmenau.de, \{dohi,nakamura,tsuruta\}@sie.dendai.ac.jp
}

\begin{abstract}
Learning systems suffer from a lack of an explicit and adaptable didactic design. A way to overcome such deficiencies is (semi-) formally representing the didactic design. A modeling approach, storyboarding, is outlined here. Storyboarding is setting the stage to apply Knowledge Engineering Technologies to verify, validate the didactics behind a learning process. As a vision, didactics can be refined according to revealed weaknesses and proven excellence. Furthermore, successful didactic patterns can be inductively inferred by analyzing the particular knowledge processing and its alleged contribution to learning success.
\end{abstract}

\section{Introduction}

Successful university instructors are often not those with the very best scientific background or outstanding research results. The most successful ones are typically those that successfully utilize didactical experiences as well as "soft skills" in dealing with other actors in the teaching process. Besides the students and colleagues, such actors include e-learning systems as well as the large amount of active (desirable and undesirable, conscious and unconscious) "content presenters" that include news, web sources and advertisements.

The design of learning activities in collegiate instruction is a very interdisciplinary process. Besides deep, topical knowledge in the subject being thought, an instructor needs knowledge and skills in many other subjects. This includes IT-related skills to use today's presentation equipment, didactic skills to effectively present the topical content, plus skills in fields like social sciences, psychology and ergonomics.

In particular, university instruction often suffers from a lack of didactic design. Since universities are also research institutions, their professors are usually hired based on their topical skills. Didactic skills are often underestimated in the recruiting process. We refrain from discussing reasons for that, but focus the issue of involving it a little more. Our approach to facing problems like these is a modeling concept for di- dactic knowledge called Storyboarding. A storyboard provides a roadmap for a course, including possible detours if certain concepts to be learned need reinforcement. Using modern media technology, a storyboard also plays the role of a server that provides the appropriate content material when deemed required. Our suggestion to ensure a wide dissemination of this concept is to use a standard tool to develop and process this model, which is Microsoft Visio.

Section 2 is an introduction to the storyboard concept. In includes the present state of the current development. This is followed by a brief introduction to an exemplary storyboard in section 3 . In section 4 , we summarize the research undertaken so far and outline current work as well as research horizons.

\section{Storyboarding}

Former Storyboarding concepts to model information and learning processes have been introduced 1998/1999 [8]. The employment of storyboarding approaches for (unfortunately, only) e-learning is characterized by misunderstandings. So-called storyboard concepts in use are mostly substitutes for softwaretechnological documents of high-level design, but are not very much specific to the instructional design process [3][14]. Didactic concepts [9] are not made explicit and, thus, pondering about didactics is not sufficiently enforced. Again, also very recent approaches as introduced above (see also [13]) remain within the borders of IT systems.

There are contrasting approaches [10] that are conceptually very useful, but syntactically much too far from a workflow directed to technology enhanced learning implementations. The crux is that purely software-technologically driven concepts do not provide an opportunity to represent and discuss details of human learning [2][4]. Learning is much more than memorizing: "Learning imposes new patterns of organization on the brain, and this phenomenon has been confirmed by electro-physiological recordings of the activity of nerve cells." ([2], p. 121). 
Learning is reasonably understood as an interactive knowledge construction process. Illustrative case studies are discussed in [5]. This book's chapter "3B Organizing Shapes" reports process of conversation and co-operation between a teacher and his students in which a variety of media types, forms of interaction, and learners' activities are dovetailed. Didactic design means the anticipation of those communication processes [9], and storyboards may provide the expressive power suitable to the design and implementation of learning processes. This, however, needs to go beyond the limits of software systems specification - the crucial question for innovations in didactic design.

Our storyboard concept is built upon standard concepts which enjoy (1) clarity by providing a highlevel modeling approach, (2) simplicity, which enables everybody to become a storyboard author, and (3) visual appearance as graphs. With respect to a better formal composition, processing, verification, validation and refinement the concept as introduced so far [7][11] has been further developed. We adopt these modifications. Here, we define a storyboard as follows:

A storyboard is a nested hierarchy of directed graphs with annotated nodes and annotated edges. Nodes are scenes or episodes. Scenes denote leaves of the nesting hierarchy. Episodes denote a sub-graph. There is exactly one Start- and End- node to each (sub) graph. Edges specify transitions between nodes. They may be single-color or bi-color. Nodes and edges have (predefined) key attributes and may have free attributes.

The interpretations of these terms follow after presenting a small example.

The representation as a graph (instead of a linear sequence) reflects the fact that different readers trace the paper differently according to their particular interests, prerequisites, a current situation (like being under time pressure), and other circumstances. The storyboard is the authors' design document representing expectations of human behavior. For exemplification, Figure 1 shows a top level storyboard on the present paper. Alternative paths may be driven by the reader's role:

- The Ilmenau research group may skip sections 1,2 and 4 , because of being familiar with it. Since the application is new to them, they study it.

- The Tokyo research group may skip sections 1 and 4. Since they are interested in the new refinements of the concept, they study section 2 . They like to know the way of storyboarding their study and therefore, study the section 3 also.

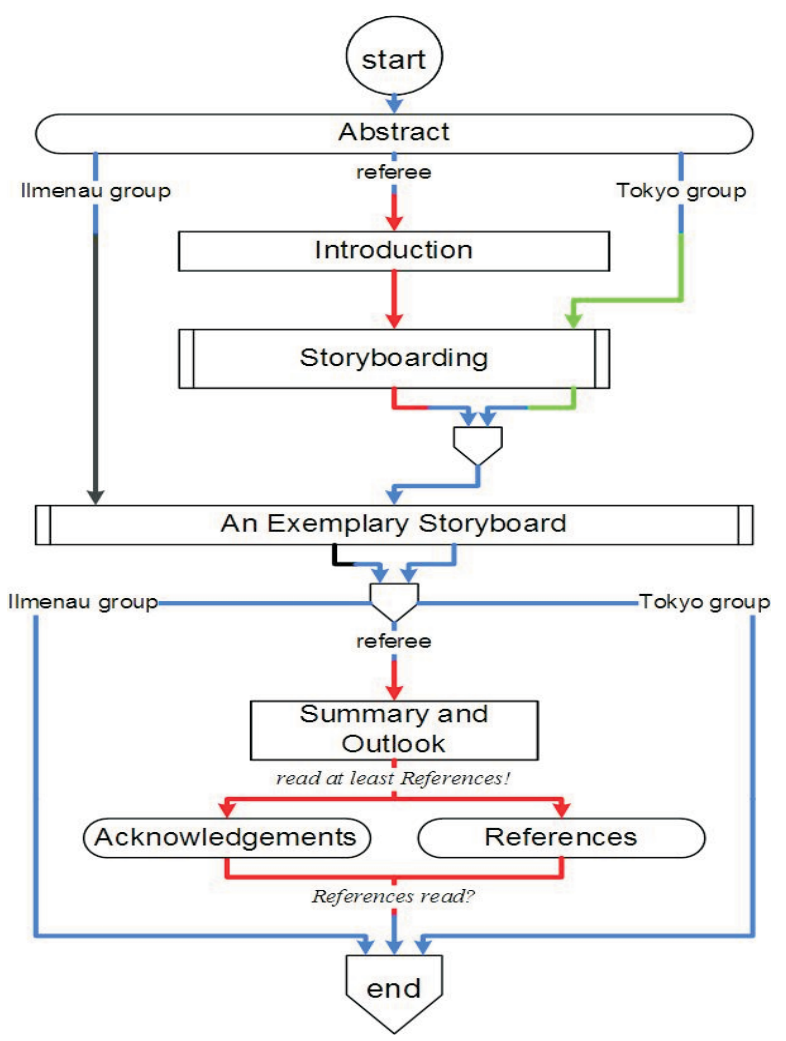

Figure 1: A Storyboard on the present paper

- Referees (hopefully) want to read all. After section 4, they can read Acknowledgements and References independently in any sequence. For their duty they have to check the References at least.

A storyboard can be traversed in different manners according to (1) users' interests, objectives, and desires, (2) didactic preferences ${ }^{1}$, (3) the sequence of nodes (and other storyboards) visited before (i.e. according to the educational history), (4) available resources (like time, money, equipment to present material, and so on) and (5) other application driven circumstances. In fact, people may read the present paper in ways that are different from our assumptions modeled in Figure 1. However, for the ways we anticipate, we can ensure that they are coherent. A storyboard may be seen as a model of an anticipated reception process that is interpreted as follows:

- Scenes denote a non-decomposable learning activity that can be implemented in any way. It can be the presentation of a (media) document, opening a tool that supports learning (URL or e-learning system) or an informal activity description.

- Episodes are defined by their sub-graph.

\footnotetext{
${ }^{1}$ In the authors' experience, some students understand better by presenting illustrations, others by providing a small example and others by providing formal descriptions.
} 
- Graphs are interpreted by the paths, on which they can be traversed.

- Start Node of a (sub-) graph defines the starting point of a legal graph traversing.

- End Node of a (sub-) graph defines the final target point of a legal graph traversing.

- Edges denote transitions between nodes. There are rules to leave a node by an outgoing edge: (1) The outgoing edge must have the same color as the incoming edge by which the node was reached. (2) If there is a condition specified as the edge's key attribute, this condition has to be met for leaving the node by this edge.

- Key attributes of nodes specify application driven information, which is necessary for all nodes of the same type, e.g. actors and locations.

- Key attributes of edges specify conditions, which have to be true for traversing on this edge.

- Free attributes specify whatever the storyboard author wants the user to know: didactic intentions, useful methods, necessary equipment, ...

Node and edge types, their visual appearance, their behavior on double click, and their behavior when following a hyperlink are as follows:

\begin{tabular}{|c|c|}
\hline Scene & \\
\hline $\begin{array}{l}\text { Behavior } \\
\text { when dou- } \\
\text { ble clicked }\end{array}$ & $\begin{array}{l}\text { - opening a document }\left({ }^{*} \cdot d o c,{ }^{*} \cdot p d f,{ }^{*} . w a v, * . v s d,\right. \\
\left.* \text { *ppt },{ }^{*} \cdot x \boldsymbol{s}, \ldots\right) \\
\text { - nothing, if just verbally described scene }\end{array}$ \\
\hline $\begin{array}{l}\text { Behavior } \\
\text { on follow- } \\
\text { ing a hy- } \\
\text { perlink }\end{array}$ & $\begin{array}{l}\text { - opening a document } \\
\text { - visiting a website with the standard browser, if it is } \\
\text { an URL } \\
\text { - opening the standard mail tool, if it is an e-mail } \\
\text { address }\end{array}$ \\
\hline
\end{tabular}

\begin{tabular}{|l|l|}
\hline Episode & \multicolumn{1}{|c|}{} \\
\hline $\begin{array}{l}\text { Behavior when } \\
\text { double clicked }\end{array}$ & $\begin{array}{l}\text { opening the sub-graph that specifies the epi- } \\
\text { sode }\end{array}$ \\
\hline $\begin{array}{l}\text { Behavior on } \\
\text { following a } \\
\text { hyperlink }\end{array}$ & $\begin{array}{l}\text { ・ opening a document } \\
\text { ・ visiting a website }\end{array}$ \\
\hline
\end{tabular}

\begin{tabular}{|l|l|}
\hline Start Node & \\
\hline $\begin{array}{l}\text { Behavior when double } \\
\text { clicked }\end{array}$ & $\begin{array}{l}\text { jumping to the Start Node of the } \\
\text { related super-graph }\end{array}$ \\
\hline $\begin{array}{l}\text { Behavior on following } \\
\text { a hyperlink }\end{array}$ & not meaningful \\
\hline
\end{tabular}

\begin{tabular}{|l|l|}
\hline End Node & End \\
\hline
\end{tabular}

\begin{tabular}{|l|l|}
\hline $\begin{array}{l}\text { Behavior when } \\
\text { double clicked }\end{array}$ & $\begin{array}{l}\text { jumping to the Reference Node that } \\
\text { successes it's associated Episode Node } \\
\text { in the related super-graph }\end{array}$ \\
\hline $\begin{array}{l}\text { Behavior on fol- } \\
\text { lowing a hyperlink }\end{array}$ & not meaningful \\
\hline
\end{tabular}

\begin{tabular}{|l|l|}
\hline Reference Node & \\
\hline $\begin{array}{l}\text { Behavior when } \\
\text { double clicked }\end{array}$ & $\begin{array}{l}\text { jumping to the End Node of the sub-graph } \\
\text { that is associated to the preceded Episode } \\
\text { Node }\end{array}$ \\
\hline $\begin{array}{l}\text { Behavior on } \\
\text { following a } \\
\text { hyperlink }\end{array}$ & not meaningful \\
\hline
\end{tabular}

The edge types are specified as follows:
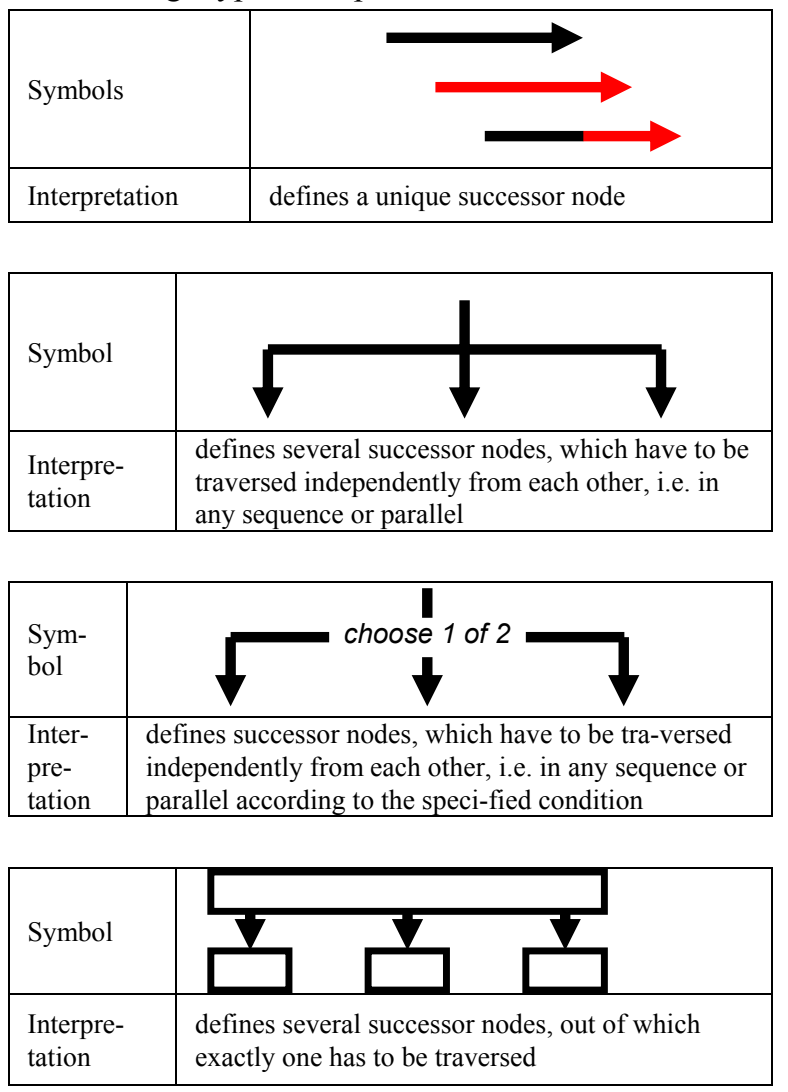

For both forking edges, there is a reverse fork at the end of the forked paths at the point of their merging. This reverse fork is marked with synchronization condition that needs to be satisfied before visiting the subsequent node.

What are peculiarities of the concept? At a first view, this purpose is similar to the purpose of traditional storyboards that are produced for shows, plays, theater games or movies, i.e. visual arts. The materials and tools of the storyboarded learning activities (e.g., text books, scripts, slides, hard- and software models, e-learning systems and others) are something compa- 
rable to the requisites of a show. Basic differences of our storyboards to those used to "specify" a show are:

- the primary purpose (learning vs. entertainment) ${ }^{2}$,

- the degree of formalization, and, as a consequence of being semi-formal,

- the obligation of everything above the level of scenes, which does (and should) not apply to storyboards in arts, in which the intendant has some freedom of individual interpretation and

- (thanks to formalization) the opportunity to formally represent, process, evaluate, and refine our storyboards, which does not apply at all to storyboards in visual arts.

Also, Storyboards have somewhat in common with classic AI knowledge representations like Semantic Networks and Frames as well as with process modeling languages like State Diagrams and Petri Nets (see e.g. [1] for use in learning processes), Workflow Diagrams (see e.g. [12] for use in learning processes) and Float Charts (see e.g. [15] for use in learning processes). Items that make this concept more expressive for didactic knowledge than representations as mentioned above are

- the potentially unlimited nesting of graphs,

- the opportunity to express "conditioned" edges by using the colors (bi-colored edges, e.g.) or respective key annotations to edges,

- the opportunity to use (two kinds of) fork-edges,

- the potential of nodes to carry many different teaching materials and tools as hyperlinks ${ }^{3}$, and, most important, and

- the fact that a scene can be implemented in any way, i.e. is not restricted to something electronically available or even formally structured (like any knowledge representations and any material included in process models).

\section{An exemplary storyboard}

For Tokyo Denki University (TDU), we developed a storyboard on the undergraduate study of Information Environment. This project is of a special interest, because TDU introduced some dynamics into the study by a system called Dynamic Learning Needs Reflection System (DLNRS) [6]. For illustration, Figure 2 shows a sub-graph behind an episode on General Cultural

\footnotetext{
2 This is no ambivalence. To include entertainment into learning is a key of successful learning and an ultimate objective of storyboarding learning processes.

${ }^{3}$ The first author developed a storyboard for an AI course at an US university and included material of his own AI course in Germany. Now, this storyboard serves both universities and is also a common platform for internationally sharing teaching materials.
}

Subjects for the undergraduate study of Information Environment at TDU.

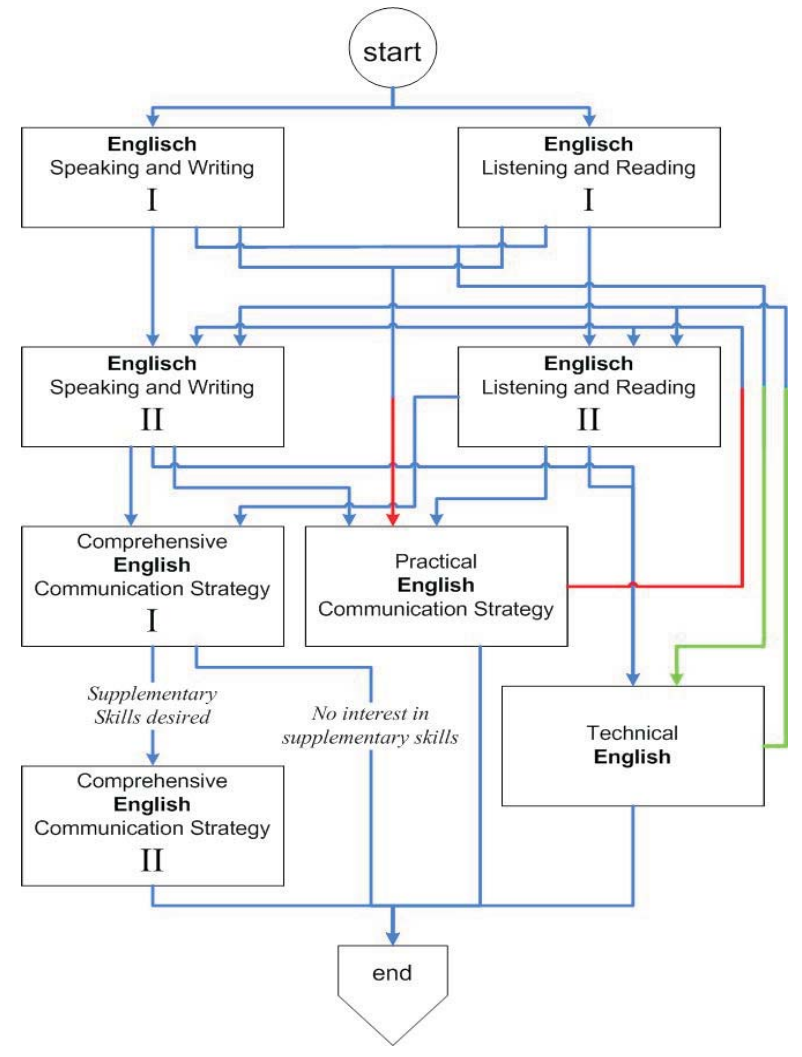

Figure 2: English language subjects at TDU

To take the individuality and dynamics into account when composing a storyboard, issues like goals, preconditions, achieved Grade Points of the previous semester and other circumstances need to be formalized and associated to both the related episodes (as a key annotation) and the students. By programming, these annotations to subjects (like their number units, e.g.) and the annotations to students (like the achieved Grade Points, e.g.) can be analyzed. This way, storyboards can be individualized and regularly (after each semester) updated according to their new status.

\section{Summary and outlook}

Storyboarding makes didactic design explicit. Since the scenes are not limited to the presentation of electronic material and represent any learning activity, this concept goes far beyond the IT approaches to support learning so far. Representing knowledge at a high level by a modeling concept that can be used by topical experts without the need of an IT- or even software technological background is very much AI-driven. To validate the usefulness in practice, we developed several storyboards on various subjects and for a complete 
university study. An essential property is simplicity in terms of both the concept itself and the tool we used to implement it. Everybody, also university instructors of subjects that are far removed from information technology, are able to develop storyboards.

Current work is dedicated to following issues: (1) We develop various particular storyboards on many different subjects, that are not limited to university teaching to derive suggestions for the refinement of the concept. (2) We introduce automated storyboard verification by structure tests like (2a) the one-to-one mapping of episode nodes and related sub-graphs, (2b) the tree structure of the graph hierarchy, (2c) the reachability of each node from the start node of its (sub-) graph, (2d) consistency in edge coloring, and (2e) certain condition checks for fork edges and their synchronization when the forked paths merge. (3) We develop a set of basic operations to compose storyboards systematically, which guarantee consistency.

Our short term objective of upcoming work is promoting the use of storyboards. As a medium term objective, we plan to develop an evaluation concept for storyboards based on the learning results of the students as acquired from the final grade they achieve for the storyboarded courses as well as the students' specific comments in a questionnaire. Our dream and long term objective is to identify typical didactic patterns of successful storyboards. Since the learning result of a particular student is associated to a particular path through the storyboard, we will be able to identify successful storyboards in general, but also successful paths within storyboards in particular. By Machine Learning methods, we'll find out what these successful storyboard paths have in common and in which properties they differ from the less successful ones. Thus, we might be able to identify successful didactic patterns. The latter is the vision of knowledge discovery in didactics. By utilizing didactic insights for the upcoming storyboards, we intend to close the loop of the never ending storyboard development spiral.

\section{References}

[1] V. Balaji, A. Arora, A. Jain, G. Goyal, G. Dubey, and S. Singh, "SCORM Learning Sequence Modeling with Petri Nets in Cooperative Learning", Learning Technology, 7(1), Special Issue on SCORM 2004 Sequencing \& Navigation, ISSN 1438-0625, 2005.

[2] J.D. Bransford, A.L. Brown, and R.R. Cocking, How People Learn: Brain, Mind, Experience, and School, National Academic Press, 2000.

[3] R.M. Briggs, L.J. Gagne, and W.W. Wager, Principles of Instructional Design, Thomson Learning, 1992.
[4] A, Damasio, The Feeling of What Happens: body and emotion in the making of consciousness, Hartcourt, 1999.

[5] B. Davis, D. Sumara, and R. Luce-Kapler, Learning and Teaching in a Complex World, Lawrence Erlbaum Associates, 2000.

[6] S. Dohi, Y. Nakamura, Y. Sakurai, S. Tsuruta, and R. Knauf, "Dynamic Learning Need Reflection System for academic education and its applicability to Intelligent Agents", IEEE International Conference on Advanced Learning Technologies (ICALT 2006), Kerkrade, Netherlands, ISBN 0-7695-2632-2, Los Alamitos, CA: IEEE Computer Society, 2006, pp. 459-463.

[7] S. Dohi, Y. Sakurai, S. Tsuruta, and R. Knauf, "Managing academic education through dynamic storyboarding", World Conference on e-Learning in Corporate, Government, Healthcare, and Higher Education 2006 (E-Learn 2006), Honolulu, Hawaii, USA, Chesapeake, VA: Association for the Advancement of Computing in Education (AACE), ISBN: 1-880094-60-6, pp. 16111619.

[8] T. Feyer and B. Thalheim, "E/R based scenario modeling for rapid prototyping of web information services", Advances in Conceptual Modeling, vol. 1727 of Lecture Notes in Computer Science (LNCS), Berlin: Springer, 1999, pp. 253-263.

[9] K.-H. Flechsig, Kleines Handbuch didaktischer Modelle\}. (German) Neuland, 1996.

[10] H.I. Forsha, The Complete Guide to Storyboarding and Problem Solving. ASQ Quality Press, 1994.

[11] K.P. Jantke and R. Knauf, "Didactic design though storyboarding: Standard concepts for standard tools", 4th International Symposium on Information and Communication Technologies, Workshop on Dissemination of e-Learning Technologies and Applications, Cape Town, South Africa, 2005, New York: ACM Press, ISBN 0-9544145-6-X, 2005, pp. 20-25.

[12] J. Lin, D. Ho, W. Sadiq, W.E. Orlowska, "Using Workflow Technology to Manage Flexible e-Learning Services", Educational Technology \& Society, 5(4), 2002.

[13] K.-D. Schewe and B. Thalheim, "The Co-Design Approach to WIS Development in E-Business and ELearning Applications", Proc. of FIPWIS 2004, Brisbane, Australia, 2004.

[14] W.J. Rothwell and H.C. Kazanas, Mastering the Instructional Design Process: A Systematic Approach. (Third Edition), Pfeiffer, 2004.

[15] E.R. Sykes and F. Franek, "A prototype for an intelligent tutoring system for students learning to program in Java", IASTED International Conference on Computers and Advanced Technology in Education, Rhodes, Greece, 2003, pp. 78. 